\title{
REFERENCES
}

1. C. E. Capel, Inverse limit spaces, Duke Math. J. vol. 21 (1954) pp. 233-245.

2. S. Eilenberg and N. E. Steenrod, Foundations of algebraic topology, Princeton University Press, 1952.

3. Hans Freudenthal, Entwicklungen von Raumen und ihrer Gruppen, Compositio Math. vol. 4 (1937) pp. 145-234.

4. J. W. T. Youngs, Homeomorphic approximations to monotone mappings, Duke Math. J. vol. 15 (1948) pp. 87-94.

UNIVERSITY OF MICHIGAN

\section{NEW METHOD FOR EXPANSION AND CONTRACTION MAPS IN UNIFORM SPACES}

\author{
THOMAS A. BROWN ${ }^{1}$ AND W. W. COMFORT ${ }^{2}$
}

1. Introduction and definitions. In [2], Freudenthal and Hurewicz showed that if the function $f$, from the totally bounded metric space $M$ onto $M$, has the property that $(f x, f y) \leqq(x, y)$ for each $x$ and $y$ in $M$, then $f$ is an isometry. By amplifying the sequential argument given in [2], Rhodes (see [4]) proved that an even stronger result holds in the more general setting of uniform spaces. Using a different method, the present paper offers a theorem similar to that of Rhodes, together with a number of results concerning "expansion" maps in uniform spaces. The notation used here, which very closely approximates that of [4], has been taken from Chapter 6 of [3].

1.1. Definition. If $(M, \mathfrak{U})$ is a uniform space, then a subset $B$ of $u$ will be called a basis for $(M, \mathfrak{u})$ if

(a) if $x \in M$ and $U \in B$, then $(x, x) \in U$;

(b) if $U \in \mathcal{U}$, then $U^{-1}$ contains a member of $B$;

(c) for each $U \in \mathcal{U}$ there is a $V \in B$ for which $V \circ V \subset U$; and

(d) for each $U \in \mathcal{U}$ and $V \in \mathcal{U}$, there is a $W \in \mathbb{B}$ for which $W \subset U \cap V$.

1.2. Definition. If $B$ is a basis for the uniform space $(M, \mathfrak{u})$, then $B$ is said to be open if each of its elements is open in $M \times M$.

1.3. Definition. If $B$ is a basis for the uniform space $(M, \mathfrak{u})$, then $B$ is said to be ample if, whenever $(x, y) \in U \in B$, there is a $W \in ß$ for which $(x, y) \in W \subset \bar{W} \subset U$.

Received by the editors September 7, 1959.

${ }^{1}$ Research conducted while a pre-doctoral fellow of the National Science Foundation.

2 Research supported by Office of Ordnance Research, U. S. Army, under contract DA-19-020-ORD-3778. 
1.4. Definition. If $(M, \mathfrak{u})$ is a uniform space with $A \subset M$ and $U \in \mathcal{U}$, then $A$ is said to be a $U$-net if $M \subset \bigcup_{a \in A} U[a]$.

1.5. Definition. A $U$-net is said to be minimal if no $U$-net has smaller cardinality.

1.6. Definition. Let $B$ be a basis for the uniform space $(M, \mathcal{u})$, and let $f$ be a function on $M$ into $M$. Then

(a) $f$ is said to be a contraction with respect to $B$ if $(f x, f y) \in U$ whenever $(x, y) \in U \in \mathbb{B}$;

(b) $f$ is said to be an expansion with respect to $B$ if $(x, y) \in U$ whenever $(f x, f y) \in U \in B$;

(c) $f$ is said to be isobasic with respect to $B$ if $f$ is both a contraction with respect to $B$ and an expansion with respect to $B$.

1.7. REMARK. If $M$ is a metric space with metric $\rho$, and if $B$ is the family of all sets of the form $U_{\epsilon}=\{(x, y) \mid \rho(x, y)<\epsilon\}$, with $\epsilon>0$, then the function $f: M \rightarrow M$ is isobasic with respect to $B$ if and only if $f$ is a $\rho$-isometry.

2.1. THEOREM. Let $B$ be an open basis for the totally bounded Hausdorff uniform space $(M, \mathcal{u})$, and let the function $f$, mapping $M$ onto $M$, be a contraction with respect to $B$. Then $(x, y) \in \bar{U}$ whenever $(f x, f y)$ $\in U \in \dot{B}$. If $B$ is ample, then $f$ is isobasic with respect to $B$.

Proof. We prove the latter of the two statements above. Given

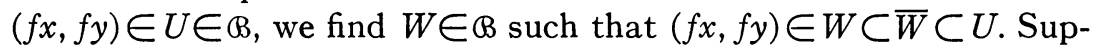
pose now that $(x, y) \notin U$. Then there is (see, for example, Theorem 7, p. 179 of [3]), a symmetric $V_{1} \in \mathcal{U}$ for which $(x, y) \notin V_{1} \circ W \circ V_{1}$. Choosing $V_{2} \in \mathcal{U}$ so that $V_{2}[f x] \times V_{2}[f y] \subset W$ and selecting $V \in \mathbb{B}$ so that $V \cup V^{-1} \subset V_{1} \cap V_{2}$, we see that if $\left(x, x^{\prime}\right) \in V$ and $\left(y, y^{\prime}\right) \in V$, then $\left(x^{\prime}, y^{\prime}\right) \notin W$ and $\left(f x^{\prime}, f y^{\prime}\right) \in W$.

Now for each minimal $V$-net $A=\left\{a_{1}, \cdots, a_{n}\right\}$ in $M$, write $(i, j) \in D(A)$ whenever $1 \leqq i<j \leqq n$ and $a_{j} \in W\left[a_{i}\right]$. Let $R(A)$ $=$ card $D(A)$, and choose $A$ to be a minimal $V$-net for which $R(A)$ is maximal. Since there is no index $i$ for which $x \in V\left[a_{i}\right]$ and $y \in V\left[a_{i}\right]$, we may suppose the notation chosen so that $x \in V\left[a_{1}\right]$ and $y \in V\left[a_{2}\right]$. Then $(1,2) \notin D(A)$. Now for $1 \leqq i \leqq n$ let $b_{i}=f a_{i}$, and let $B=\left\{b_{1}, \cdots, b_{n}\right\}$. Then $B$ is a minimal $V$-net, and $(1,2) \in D(B)$. But $(i, j) \in D(B)$ whenever $(i, j) \in D(A)$, so that $R(B)>R(A)$. This contradiction completes the proof.

2.2. Corollary. Edrei (See Theorem 1 of [1]). Let $M$ be a totally bounded metric space and let the function $f$ map $M$ onto $M$. Suppose that there exists $\epsilon>0$ for which $(f x, f y)<\epsilon$ whenever $(x, y)<\epsilon$. Then $(f x, f y)=(x, y)$ whenever $(x, y)<\epsilon$. 
3. Expansion maps in uniform spaces. In this section we show, by employing a device similar to that of Theorem 2.1, that an expansion map on a compact Hausdorff space into itself must be an onto mapping.

3.1. Proposition. Let the uniform space $(M, \mathfrak{u})$ be compact Hausdorff, and let $B$ be a basis for $(M, \mathcal{u})$. Let $f: M \rightarrow M$ be an expansion with respect to $B$, and suppose that $f\left(x_{\alpha}\right)$ and $f\left(y_{\beta}\right)$ are nets in $f M$ which converge to a common point. Then the nets $x_{\alpha}, y_{\beta}$ converge to a common point.

3.2. Lemma. Let the uniform space $(M, \mathfrak{u})$ be totally bounded Hausdorff, and let $\mathrm{B}$ be a basis for $(M, \mathfrak{u})$. Let $f: M \rightarrow M$ be an expansion with respect to $B$. Then $f M$ is dense in $M$.

Proof. Suppose that there exist $p \in M$ and $U \in B$ for which $U[p] \subset M \backslash f M$. For each subset $F$ of $M$, write $F \in \mathcal{F}$ if $(x, y) \in U$ whenever $x \in F$ and $y \in F$. The family $\mathcal{F}$ admits a finite subcover of $M$, since if $V \in \Theta$ and $V \circ V \subset U$, then \{int $V[x] \mid x \in M\}$ covers $M$. Let $\left\{F_{1}, \cdots, F_{k}\right\}$ be a subset of $\mathcal{F}$ which covers $M$, selected so that $k$ is minimal. We may suppose that $p \in F_{k}$. Then $F_{k} \subset U[p]$, so that $f^{-1} F_{k}=\Lambda$. If $1 \leqq i \leqq k-1$, then $f^{-1} F_{i} \in \mathcal{F} ;$ and $\bigcup_{i=1}^{k-1} f^{-1} F_{i}=M$, contrary to the minimality of $k$.

3.3. Definition. Let the uniform space $(M, \mathfrak{u})$ be compact Hausdorff, and let $B$ be a basis for $(M, \mathcal{u})$. Let $f: M \rightarrow M$ be an expansion with respect to $B$, and suppose that $f\left(x_{\alpha}\right)$ is a net in $f M$ which approaches the point $p \in M$. Then the limit in $M$ of the net $x_{\alpha}$ is called the pseudo-inverse (under $f$ ) of $p$, and is denoted $p^{*}$.

3.4. Proposition. Let $M, \mathcal{U}, \Theta$ and $f$ be as in 3.3, and suppose that $B$ is ample. Then $\left(x^{*}, y^{*}\right) \in U$ whenever $(x, y) \in U \in B$.

3.5. Theorem. Let the uniform space $(M, \mathcal{u})$ be compact Hausdorff, and let $B$ be an ample basis for $(M, \mathcal{u})$. Let $f: M \rightarrow M$ be an expansion with respect to $B$. Then $f\left(p^{*}\right)=p$ for each $p \in M$, so that $f M=M$.

Proof. If there is a $p \in M$ for which $f\left(p^{*}\right) \neq p$, then there is a $U \in \beta$ for which $\left(f\left(p^{*}\right), p\right) \notin U$. We select $V \in \beta$ so that $V \circ V^{-1}$ $\circ V \circ V^{-1} \subset U$.

Now for each minimal $V$-net $A=\left\{a_{1}, \cdots, a_{n}\right\}$ in $M$, write $(i, j) \in E(A)$ whenever $1 \leqq i<j \leqq n$ and $V\left[a_{i}\right] \cap V\left[a_{j}\right] \neq \Lambda$. Let $S(A)$ $=$ card $E(A)$, and choose $A$ to be a minimal $V$-net for which $S(A)$ is maximal. Since there is no index $i$ for which $p \in V\left[a_{i}\right]$ and $f\left(p^{*}\right)$ $\in V\left[a_{i}\right]$, we may suppose the notation chosen so that $p \in V\left[a_{1}\right]$ and 
$f\left(p^{*}\right) \in V\left[a_{2}\right]$. Then $(1,2) \notin E(A)$. Let $B=\left\{a_{1}^{*}, \cdots, a_{n}^{*}\right\}$. Since $x=(f x)^{*}$ for each $x \in M$, it follows from 3.4 that $B$ is a minimal $V$-net in $M$ and that $p \in V\left[a_{1}^{*}\right] \cap V\left[a_{2}^{*}\right]$. Hence $(1,2) \in E(B)$. But $(i, j)$ $\in E(B)$ whenever $(i, j) \in E(A)$, so that $S(B)>S(A)$. This contradiction completes the proof.

3.6. Corollary. Banach-Ulam (See Theorem 3 of [5]). A compact metric space cannot be isometric with a proper subset of itself.

3.7. Corollary. Let the uniform space $(M, \mathcal{u})$ be compact Hausdorff, and let $\mathrm{B}$ be an ample, open basis for $(M, \mathfrak{u})$. Let $f: M \rightarrow M$ be an expansion with respect to $B$. Then $f$ is isobasic with respect to $B$.

Proof. The function $f$ is clearly (1-1), and its inverse function $f^{-1}$ exists by 3.5. Since $f^{-1}$ satisfies the hypotheses of $2.1, f^{-1}$ is isobasic with respect to $B$. Hence $f$ is also.

3.8. Corollary. Let $M$ be a compact metric space and let $f: M \rightarrow M$. Suppose that there exists $\epsilon>0$ for which $(f x, f y) \geqq(x, y)$ whenever $(x, y)<\epsilon$. Then $(f x, f y)=(x, y)$ whenever $(x, y)<\epsilon$.

\section{REFERENCES}

1. Albert Edrei, On mappings which do not increase small distances, Proc. London Math. Soc. (3) vol. 2 (1952) pp. 272-278.

2. H. Freudenthal and W. Hurewicz, Dehnungen, Verkurzungen, Isometrien, Fund. Math. vol. 26 (1936) pp. 120-122.

3. John L. Kelley, General topology, New York, Van Nostrand, 1955.

4. F. Rhodes, $A$ generalization of isometries to uniform spaces, Proc. Cambridge Philos. Soc. vol. 52 (1956) pp. 399-405.

5. S. Ulam, The Scottish book of Lwow, mimeographed.

HARVARD UNIVERSITY 